\title{
THE REACTION OF THE CAPILLARY BED OF THE NAILFOLD TO THE CONTINU- OUS INTRAVENOUS INFUSION OF LEVO-NOR-EPINEPHRINE IN PATIENTS WITH NORMAL BLOOD PRESSURE AND WITH ESSENTIAL HYPERTENSION
}

\author{
By SHELDON E. GREISMAN \\ (From the Department of Medicine, New York University College of Medicine and the Third \\ (New York University) Medical Division, Bellevue Hospital, New York, N. Y.)
}

(Submitted for publication June 12, 1953; accepted March 3, 1954)

The intravenous infusion of 1-nor-epinephrine in persons with normal cardiovascular systems produces a hemodynamic state which in many respects resembles that found in patients with essential hypertension. In the systemic circulation there is in each of the two conditions an elevation of the systolic, diastolic and mean arterial pressures, an unchanged or moderately decreased cardiac output, an increase in systemic peripheral resistance and usually an absence of subjective symptoms (1). This similarity in the two hemodynamic states has led to the suggestion that l-nor-epinephrine may be a humoral agent responsible for essential hypertension. If this were true, then the hypertensive state produced by 1-nor-epinephrine should produce changes in all portions of the circulatory system similar to those found in essential hypertension. The dynamics of the pulmonary circulation have been shown to be different in the two states; the intravenous infusion of 1-nor-epinephrine raises mean pulmonary arterial pressure whereas this pressure is normal in patients with uncomplicated essential hypertension (1).

The present study was undertaken to determine whether the circulation in specific portions of the systemic vascular system, in this instance the peripheral cutaneous capillaries, is the same in subjects with 1-nor-epinephrine induced hypertension as in patients with essential hypertension. Accordingly, the capillary circulation of the nailfold during the sustained hypertensive state induced in control subjects by continuous intravenous infusion of 1-nor-epinephrine was compared with the nailfold capillary circulation of patients with essential hypertension. The observations indicate that for similar levels of elevated arterial pressure the capillary circulation of the nailfold is more reactive in the l-nor-epinephrine induced hypertension than in essential hypertension. A previous study indicated that the nailfold capillary circulation of subjects with essential hypertension was more reactive to single rapid injections of epinephrine than the similar capillary circulation of control subjects (2). The present study extends this observation to indicate that such hyper-reactivity persists over the several hours of a continuous infusion of 1-nor-epinephrine.

\section{METHOD}

Two groups of hospitalized patients were selected; one with normal cardiovascular systems, the other with essential hypertension. The hypertensive patients had known essential hypertension for many years or had been followed on the wards for several weeks and had generally maintained diastolic blood pressure levels of $90 \mathrm{~mm}$. $\mathrm{Hg}$ or higher. Patients receiving medication which could produce cardiovascular changes were excluded. An attempt was made to select patients of relatively similar ages in the two groups. During the observations the subjects were recumbent with a light blanket over the trunk and lower extremities. Patients with cool, pale, or cyanotic fingers were not studied. One arm was abducted in the horizontal plane approximately thirty degrees from the side, with the terminal phalanx of the fourth finger at about the level of the sternum. The finger was steadied by a clamp applied along the lateral margins of the phalanx. Cedar oil was applied to the base of the nail bed and the terminal capillary loops were then readily visualized microscopically at $96 \times$ when illuminated by a slit lamp. A blood pressure cuff was placed about the opposite arm and an infusion of isotonic salt solution was begun in an antecubital vein in such a manner that there was no interference with auscultatory blood pressure determinations. Following these procedures twenty minutes were allowed to elapse, or until the blood pressure had stabilized. If at the end of this period the fingers had become cool, pale, or cyanotic, or if the blood flow through the capillary loops had slowed, as visualized microscopically, another twenty minutes were allowed to elapse. If the fingers did not become warm and the capillary blood flow did not become rapid, no observations were made. After the 
TABLE I

Alteration of blood pressure, pulse rate, and vasomotion of the capillary bed of the fingernail during l-nor-epinephrine infusion in patients with normal blood pressure

\begin{tabular}{|c|c|c|c|c|c|c|c|c|}
\hline $\begin{array}{l}\text { Patient } \\
\text { no. }\end{array}$ & Age & Sex & Diagnosis & $\begin{array}{c}\text { Rate of } \\
\text { norepinephrine } \\
\text { infusion } \\
(\text { gamma/Kg./minute })\end{array}$ & $\begin{array}{l}\text { Arterial } \\
\text { blood } \\
\text { pressure } \\
(\mathrm{mm} . \mathrm{Hg})\end{array}$ & $\begin{array}{l}\text { Pulse } \\
\text { rate } \\
(\text { per } \\
\text { minute })\end{array}$ & $\begin{array}{c}\text { Intensity of } \\
\text { vasomotion of } \\
\text { the capillary bed } \\
\text { of nailfold* }\end{array}$ & $\begin{array}{l}\text { Duration } \\
\text { of } \\
\text { infusion } \\
\text { (minutes) }\end{array}$ \\
\hline 1 & 56 & F & $\begin{array}{l}\text { Pulmonary } \\
\text { fibrosis and } \\
\text { emphysema }\end{array}$ & $\begin{array}{l}-.05 \\
.05 \\
.10 \\
.17 \\
.23 \\
.30 \\
.32\end{array}$ & $\begin{array}{l}110 / 54 \\
120 / 56 \\
118 / 60 \\
146 / 72 \\
170 / 80 \\
180 / 80 \\
180 / 80\end{array}$ & $\begin{array}{l}84 \\
84 \\
80 \\
74 \\
72 \\
72 \\
60\end{array}$ & $\begin{array}{c}0 \\
0 \\
\times \\
2 \times \\
3 \times \\
\text { Initial ischemia } \\
\text { Ischemia }\end{array}$ & $\begin{array}{r}- \\
5 \\
5 \\
10 \\
10 \\
10 \\
40\end{array}$ \\
\hline 2 & 50 & $\mathrm{M}$ & $\begin{array}{l}\text { Recovered } \\
\text { barbiturate } \\
\text { intoxication }\end{array}$ & $\begin{array}{l}-\overline{.} \\
.03 \\
.06 \\
.07 \\
.09 \\
.13 \\
.17 \\
.26 \\
.29\end{array}$ & $\begin{array}{l}106 / 58 \\
106 / 58 \\
106 / 58 \\
108 / 64 \\
112 / 68 \\
120 / 76 \\
130 / 80 \\
140 / 84 \\
150 / 90\end{array}$ & $\begin{array}{l}72 \\
72 \\
72 \\
72 \\
70 \\
68 \\
68 \\
64 \\
58\end{array}$ & $\begin{array}{c}1 \times \\
1 \times \\
1 \times \\
2 \times \\
2 \times \\
3 \times \\
\text { Initial ischemia } \\
\text { Ischemia } \\
\text { Ischemia }\end{array}$ & $\begin{array}{r}- \\
5 \\
5 \\
10 \\
5 \\
10 \\
15 \\
15 \\
30\end{array}$ \\
\hline 3 & 35 & $M$ & $\begin{array}{l}\text { Normal } \\
\text { male }\end{array}$ & $\begin{array}{l}-. \\
.12 \\
.15\end{array}$ & $\begin{array}{l}110 / 60 \\
120 / 74 \\
120 / 74\end{array}$ & $\begin{array}{l}74 \\
64 \\
64\end{array}$ & $\begin{array}{c}\stackrel{x}{3 \times} \\
\text { Initial ischemia }\end{array}$ & $\begin{array}{l}-\overline{10} \\
20\end{array}$ \\
\hline 4 & 56 & $\mathrm{M}$ & $\begin{array}{l}\text { Chronic } \\
\text { alcoholism }\end{array}$ & $\begin{array}{l}-\overline{.03} \\
.21 \\
.30 \\
.52\end{array}$ & $\begin{array}{l}114 / 60 \\
114 / 62 \\
130 / 70 \\
150 / 80 \\
160 / 80\end{array}$ & $\begin{array}{l}72 \\
72 \\
60 \\
56 \\
48\end{array}$ & $\begin{array}{c}\stackrel{\times}{1 \times} \\
3 \times \\
\text { Initial ischemia } \\
\text { Ischemia }\end{array}$ & $\begin{array}{r}- \\
5 \\
10 \\
20 \\
25\end{array}$ \\
\hline 5 & 27 & $\mathrm{M}$ & $\begin{array}{l}\text { Pharyngitis } \\
\text { (convalescent) }\end{array}$ & $\begin{array}{l}-.02 \\
.06 \\
.10 \\
.12 \\
.20 \\
.25 \\
.31\end{array}$ & $\begin{array}{l}126 / 60 \\
120 / 60 \\
126 / 60 \\
124 / 64 \\
126 / 70 \\
130 / 70 \\
136 / 74 \\
140 / 70\end{array}$ & $\begin{array}{l}76 \\
76 \\
76 \\
76 \\
76 \\
74 \\
72 \\
70\end{array}$ & $\begin{array}{c}1 \times \\
1 \times \\
1 \times \\
2 \times \\
2 \times \\
\text { Initial ischemia } \\
\text { Ischemia } \\
\text { Ischemia }\end{array}$ & $\begin{array}{r}- \\
5 \\
5 \\
10 \\
10 \\
10 \\
10 \\
20\end{array}$ \\
\hline 6 & 47 & $F$ & $\begin{array}{l}\text { Barbiturate } \\
\text { addiction }\end{array}$ & $\begin{array}{l}-. \\
.08 \\
.20 \\
.32\end{array}$ & $\begin{array}{l}100 / 70 \\
108 / 74 \\
120 / 80 \\
140 / 88\end{array}$ & $\begin{array}{l}76 \\
76 \\
72 \\
70\end{array}$ & $\begin{array}{c}\stackrel{x}{x} \\
2 \underset{x}{\text { Initial ischemia }}\end{array}$ & $\begin{array}{l}\overline{10} \\
10 \\
30\end{array}$ \\
\hline 7 a) & 38 & $F$ & $\begin{array}{l}\text { Chronic } \\
\text { alcoholism }\end{array}$ & $\begin{array}{l}-\overline{.04} \\
.11 \\
.22\end{array}$ & $\begin{array}{l}110 / 60 \\
110 / 60 \\
130 / 76 \\
140 / 88\end{array}$ & $\begin{array}{l}84 \\
84 \\
84 \\
84\end{array}$ & $\begin{array}{c}1 \times \\
2 \times \\
3 \times \\
\text { Initial ischemia }\end{array}$ & $\begin{array}{l}\overline{15} \\
15 \\
75\end{array}$ \\
\hline b) & & & $\begin{array}{l}\text { Same patient } \\
3 \text { days later }\end{array}$ & $\overline{.22}$ & $\begin{array}{l}110 / 70 \\
140 / 88\end{array}$ & $\begin{array}{l}84 \\
72\end{array}$ & $\stackrel{1 \times}{\text { Initial ischemia }}$ & $\overline{180}$ \\
\hline 8 & 22 & $F$ & $\begin{array}{l}\text { Normal } \\
\text { female }\end{array}$ & $\begin{array}{l}-\overline{.05} \\
.09 \\
.13\end{array}$ & $\begin{array}{l}120 / 70 \\
120 / 70 \\
126 / 80 \\
130 / 90\end{array}$ & $\begin{array}{l}72 \\
72 \\
72 \\
66\end{array}$ & $\begin{array}{c}0 \\
1 \times \\
2 \times \\
\text { Initial ischemia }\end{array}$ & $\begin{array}{r}- \\
5 \\
10 \\
20\end{array}$ \\
\hline 9 a) & 74 & $\mathbf{M}$ & $\begin{array}{l}\text { Esophageal } \\
\text { carcinoma }\end{array}$ & $\begin{array}{l}-\overline{.04} \\
.06 \\
.08 \\
.12 \\
.28\end{array}$ & $\begin{array}{l}154 / 70 \\
150 / 70 \\
150 / 72 \\
180 / 84 \\
200 / 88 \\
220 / 100\end{array}$ & $\begin{array}{l}80 \\
80 \\
80 \\
76 \\
74 \\
70\end{array}$ & $\begin{array}{c}\times x \\
\times \\
\times \\
2 \times \\
3 \times \\
\text { Initial ischemia }\end{array}$ & $\begin{array}{r}- \\
5 \\
5 \\
10 \\
10 \\
30\end{array}$ \\
\hline
\end{tabular}

* Average number of constrictions of the arterial segments of the capillary loops per minute. $0=0-2, \times=3-8$, $1 \times=9-14,2 \times=15-25,3 \times=25-30$. 


\begin{tabular}{|c|c|c|c|c|c|c|c|c|}
\hline $\begin{array}{l}\text { Patient } \\
\text { no. }\end{array}$ & Age & Sex & Diagnosis & $\begin{array}{c}\text { Rate of } \\
\text { norepinephrine } \\
\text { infusion } \\
(\text { gamma } / \text { Kg. } / \text { minute })\end{array}$ & $\begin{array}{l}\text { Arterial } \\
\text { blood } \\
\text { pressure } \\
(m m . H g)\end{array}$ & $\begin{array}{l}\text { Pulse } \\
\text { rate } \\
\text { (per } \\
\text { minute) }\end{array}$ & $\begin{array}{l}\text { Intensity of } \\
\text { vasomotion of } \\
\text { the capillary bed } \\
\text { of nailfold* }\end{array}$ & $\begin{array}{c}\text { Duration } \\
\text { of } \\
\text { infusion } \\
\text { (minutes) }\end{array}$ \\
\hline $9 \mathrm{~b})$ & & & $\begin{array}{l}\text { Same patient } \\
5 \text { days later }\end{array}$ & $\begin{array}{l}.03 \\
.06 \\
.10 \\
.20\end{array}$ & $\begin{array}{l}130 / 70 \\
130 / 70 \\
140 / 76 \\
170 / 80 \\
180 / 90\end{array}$ & $\begin{array}{l}72 \\
72 \\
72 \\
66 \\
64\end{array}$ & 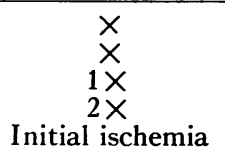 & $\begin{array}{r}- \\
5 \\
5 \\
5 \\
25\end{array}$ \\
\hline 10 & 33 & $\mathrm{~F}$ & $\begin{array}{l}\text { Recovered } \\
\text { barbiturate } \\
\text { intoxication }\end{array}$ & $\begin{array}{l}-.02 \\
.07 \\
.10 \\
.13 \\
.17 \\
.20\end{array}$ & $\begin{array}{l}116 / 76 \\
116 / 76 \\
120 / 78 \\
120 / 80 \\
130 / 86 \\
146 / 86 \\
146 / 86\end{array}$ & $\begin{array}{l}92 \\
92 \\
92 \\
92 \\
90 \\
88 \\
84\end{array}$ & $\begin{array}{c}1 \times \\
1 \times \\
2 \times \\
3 \times \\
\text { Initial ischemia } \\
\text { Ischemia } \\
\text { Ischemia }\end{array}$ & $\begin{array}{r}- \\
5 \\
5 \\
5 \\
5 \\
5 \\
120\end{array}$ \\
\hline 11 & 67 & $\mathbf{M}$ & $\begin{array}{l}\text { Resolved } \\
\text { broncho } \\
\text { pneumonia }\end{array}$ & $\begin{array}{l}\overline{.06} \\
.12 \\
.24 \\
.37 \\
.48\end{array}$ & $\begin{array}{l}140 / 76 \\
150 / 80 \\
160 / 80 \\
190 / 88 \\
220 / 90 \\
240 / 100\end{array}$ & $\begin{array}{l}76 \\
76 \\
74 \\
68 \\
60 \\
56\end{array}$ & $\begin{array}{c}1 \times \\
2 \times \\
3 \times \\
\text { Initial ischemia } \\
\text { Ischemia } \\
\text { Ischemia }\end{array}$ & $\begin{array}{r}- \\
5 \\
5 \\
10 \\
15 \\
20\end{array}$ \\
\hline 12 & 36 & $F$ & $\begin{array}{l}\text { Chronic } \\
\text { alcoholism }\end{array}$ & $\begin{array}{l}-. \\
.09 \\
.17 \\
.22 \\
.26 \\
.34\end{array}$ & $\begin{array}{l}130 / 80 \\
130 / 84 \\
150 / 90 \\
150 / 94 \\
160 / 100 \\
170 / 110\end{array}$ & $\begin{array}{l}88 \\
84 \\
72 \\
68 \\
60 \\
56\end{array}$ & $\begin{array}{c}2 \times \\
3 \times \\
\text { Initial ischemia } \\
\text { Ischemia } \\
\text { Ischemia } \\
\text { Ischemia }\end{array}$ & $\begin{array}{r}- \\
5 \\
10 \\
10 \\
15 \\
25\end{array}$ \\
\hline 13 & 77 & $\mathbf{M}$ & Chronic gout & $\begin{array}{l}.07 \\
.07 \\
.17 \\
.27\end{array}$ & $\begin{array}{l}140 / 80 \\
150 / 86 \\
180 / 86 \\
190 / 86 \\
220 / 96\end{array}$ & $\begin{array}{l}84 \\
84 \\
80 \\
76 \\
72\end{array}$ & $\begin{array}{c}\underset{1}{\times} \\
2 \times \\
3 \times \\
\text { Initial ischemia }\end{array}$ & $\begin{array}{r}- \\
5 \\
5 \\
10 \\
60\end{array}$ \\
\hline 14 & 53 & $\mathrm{~F}$ & $\begin{array}{l}\text { Recovered } \\
\text { barbiturate } \\
\text { intoxication }\end{array}$ & $\begin{array}{l}- \\
.19 \\
.33 \\
.40\end{array}$ & $\begin{array}{l}142 / 80 \\
190 / 94 \\
190 / 98 \\
220 / 112\end{array}$ & $\begin{array}{l}72 \\
68 \\
60 \\
56\end{array}$ & $\begin{array}{c}\text { X } \\
\text { Initial ischemia } \\
\text { Ischemia }\end{array}$ & $\begin{array}{r}-5 \\
15 \\
25\end{array}$ \\
\hline 15 & 63 & $\mathbf{M}$ & $\begin{array}{l}\text { Pulmonary } \\
\text { fibrosis and } \\
\text { emphysema }\end{array}$ & $\begin{array}{l}-16 \\
.16 \\
.20 \\
.40\end{array}$ & $\begin{array}{l}130 / 90 \\
186 / 108 \\
212 / 116 \\
240 / 120\end{array}$ & $\begin{array}{l}72 \\
72 \\
70 \\
70\end{array}$ & $\begin{array}{c}1 \times \\
2 \times \\
3 \times \\
\text { Initial ischemia }\end{array}$ & $\begin{array}{r}-5 \\
10 \\
30\end{array}$ \\
\hline
\end{tabular}

control period a solution of 1-nor-epinephrine, 14 gamma per cubic centimeter in isotonic saline solution, was then substituted for the original saline solution, without disturbing the patient, by changing the infusion bottle. The rate of infusion of the 1-nor-epinephrine was increased progressively and with each increment the arterial blood pressure, the pulse rate, and the reaction of the terminal capillary loops at the base of the fingernail were recorded. The rates and duration of infusion of 1-nor-epinephrine are indicated in Tables I and II.

\section{RESULTS}

\section{Reactivity of capillary bed}

The terminal capillary loops of the nailfold of the finger were usually patent with a rapid blood

1 Bitartrate salt (synthetic: supplied by WinthropStearns, Inc.). flow in both normotensive and hypertensive subjects. Intermittent momentary interruptions of blood flow occurred in the arterial segments of the capillary loops. The cause of these interruptions in blood flow could not be determined directly since the vascular bed proximal to the terminal capillary loops could not be visualized clearly. However, when the capillary beds of human bulbar conjunctivae and of the mesenteries of mice were observed, similar, though less frequent interruptions in capillary blood flow were seen. These gaps in blood flow were observed to be produced by intermittent constriction of the metarterioles and precapillary segments of these vascular beds and epinephrine was seen to increase the frequency of constriction of these metarterioles and precapil- 
TABLE II

Alteration of blood pressure, pulse rate, and vasomotion of the capillary bed of the fingernail during l-nor-epinephrine infusion in patients with essential hypertension

\begin{tabular}{|c|c|c|c|c|c|c|c|c|}
\hline $\begin{array}{l}\text { Patient } \\
\text { no. }\end{array}$ & Age & Sex & Diagnosis & $\begin{array}{c}\text { Rate of } \\
\text { norepinephrine } \\
\text { infusion } \\
(\text { gamma } / K g . / m i n u t e)\end{array}$ & $\begin{array}{l}\text { Arterial } \\
\text { blood } \\
\text { pressure } \\
(m m . H g)\end{array}$ & $\begin{array}{l}\text { Pulse } \\
\text { rate } \\
\text { (per } \\
\text { minute) }\end{array}$ & $\begin{array}{l}\text { Intensity of } \\
\text { vasomotion of } \\
\text { the capillary bed } \\
\text { of nailfold* }\end{array}$ & $\begin{array}{c}\text { Duration } \\
\text { of } \\
\text { infusion } \\
\text { (minutes) }\end{array}$ \\
\hline 1 & 46 & $\mathrm{~F}$ & $\begin{array}{l}\text { Hypertensive } \\
\text { vascular } \\
\text { disease } \\
\text { (known } 10 \text { years) }\end{array}$ & $\begin{array}{l}-\overline{.04} \\
.07 \\
.13 \\
.16\end{array}$ & $\begin{array}{l}146 / 86 \\
160 / 90 \\
172 / 98 \\
184 / 104 \\
190 / 110\end{array}$ & $\begin{array}{l}72 \\
72 \\
72 \\
72 \\
70\end{array}$ & $\begin{array}{c}2 \times \\
2 \times \\
3 \times \\
\text { Initial ischemia } \\
\text { Ischemia }\end{array}$ & $\begin{array}{l}- \\
10 \\
10 \\
10 \\
10\end{array}$ \\
\hline 2 & 68 & $\mathrm{~F}$ & $\begin{array}{l}\text { 1) Hypertensive } \\
\text { vascular } \\
\text { disease } \\
\text { (unknown } \\
\text { duration) } \\
\text { 2) Old cerebro- } \\
\text { vascular } \\
\text { accident }\end{array}$ & $\begin{array}{l}-\overline{.06} \\
.11 \\
.16 \\
.26\end{array}$ & $\begin{array}{l}154 / 88 \\
160 / 88 \\
166 / 94 \\
170 / 96 \\
188 / 98\end{array}$ & $\begin{array}{l}88 \\
88 \\
88 \\
88 \\
88\end{array}$ & $\begin{array}{c}2 \times \\
3 \times \\
\text { Initial ischemia } \\
\text { Ischemia } \\
\text { Ischemia }\end{array}$ & $\begin{array}{l}- \\
10 \\
15 \\
15 \\
15\end{array}$ \\
\hline 3 & 54 & $\mathrm{~F}$ & $\begin{array}{l}\text { 1) Hypertensive } \\
\text { vascular } \\
\text { disease } \\
\text { (known 11 years) } \\
\text { 2) Hypertensive } \\
\text { cardiovascular } \\
\text { disease II B }\end{array}$ & $\begin{array}{l}-\overline{.04} \\
.07 \\
.10 \\
.13\end{array}$ & $\begin{array}{l}170 / 86 \\
170 / 86 \\
190 / 86 \\
212 / 96 \\
230 / 96\end{array}$ & $\begin{array}{l}60 \\
60 \\
51 \\
48 \\
45\end{array}$ & $\begin{array}{c}1 \times \\
3 \times \\
\text { Initial ischemia } \\
\text { Ischemia } \\
\text { Ischemia }\end{array}$ & $\begin{array}{l}-15 \\
15 \\
15 \\
15\end{array}$ \\
\hline 4 & 52 & $\mathrm{~F}$ & $\begin{array}{l}\text { 1) Hypertensive } \\
\text { vascular } \\
\text { disease } \\
\text { (known 9 years) } \\
\text { 2) Old myocardial } \\
\text { infarction }\end{array}$ & $\begin{array}{l}\overline{.05} \\
.08 \\
.17\end{array}$ & $\begin{array}{l}150 / 90 \\
150 / 90 \\
170 / 98 \\
200 / 108\end{array}$ & $\begin{array}{l}92 \\
88 \\
88 \\
86\end{array}$ & $\begin{array}{c}2 \times \\
3 \times \\
\text { Initial ischemia } \\
\text { Ischemia }\end{array}$ & $\begin{array}{l}-15 \\
15 \\
10\end{array}$ \\
\hline 5 & 60 & $\mathbf{M}$ & $\begin{array}{l}\text { 1) Hypertensive } \\
\text { vascular } \\
\text { disease } \\
\text { (unknown } \\
\text { duration) } \\
\text { 2) Cerebral } \\
\text { arteriosclerosis }\end{array}$ & $\begin{array}{l}\overline{.05} \\
.11 \\
.21\end{array}$ & $\begin{array}{l}190 / 90 \\
194 / 90 \\
210 / 90 \\
240 / 110\end{array}$ & $\begin{array}{l}72 \\
72 \\
60 \\
57\end{array}$ & $\begin{array}{c}3 \times \\
\text { Initial ischemia } \\
\text { Ischemia } \\
\text { Ischemia }\end{array}$ & $\begin{array}{l}\overline{10} \\
10 \\
10\end{array}$ \\
\hline 6 & 73 & $F$ & $\begin{array}{l}\text { 1) Hypertensive } \\
\text { vascular } \\
\text { disease } \\
\text { (known } 15 \text { years) } \\
\text { 2) Old cerebro- } \\
\text { vascular } \\
\text { accident }\end{array}$ & $\begin{array}{l}-\overline{.05} \\
.09 \\
.18 \\
.25\end{array}$ & $\begin{array}{l}190 / 94 \\
198 / 100 \\
208 / 100 \\
230 / 120 \\
238 / 120\end{array}$ & $\begin{array}{r}100 \\
96 \\
100 \\
96 \\
84\end{array}$ & $\begin{array}{l}2 \times \\
\text { Initial ischemia } \\
\text { Ischemia } \\
\text { Ischemia } \\
\text { Ischemia }\end{array}$ & $\begin{array}{l}\overline{15} \\
10 \\
10 \\
10\end{array}$ \\
\hline 7 & 67 & $\mathrm{~F}$ & $\begin{array}{l}\text { Hypertensive } \\
\text { vascular } \\
\text { disease } \\
\text { (known } 10 \text { years) }\end{array}$ & $\begin{array}{l}-\overline{.06} \\
.09 \\
.16\end{array}$ & $\begin{array}{l}220 / 94 \\
240 / 90 \\
248 / 94 \\
284 / 94\end{array}$ & $\begin{array}{l}84 \\
80 \\
84 \\
84\end{array}$ & $\begin{array}{c}2 \times \\
2 \times \\
\text { Initial ischemia } \\
\text { Ischemia }\end{array}$ & $\begin{array}{l}- \\
10 \\
15 \\
10\end{array}$ \\
\hline 8 & 71 & $\mathrm{~F}$ & $\begin{array}{l}\text { 1) Hypertensive } \\
\text { vascular } \\
\text { disease } \\
\text { (unknown } \\
\text { duration) } \\
\text { 2) Bronchogenic } \\
\text { carcinoma } \\
\end{array}$ & $\begin{array}{l}.07 \\
.22 \\
.31 \\
.71\end{array}$ & $\begin{array}{l}220 / 100 \\
220 / 106 \\
220 / 110 \\
240 / 120 \\
240 / 120\end{array}$ & $\begin{array}{l}88 \\
88 \\
80 \\
80 \\
76\end{array}$ & $\begin{array}{c}3 \times \\
\text { Initial ischemia } \\
\text { Ischemia } \\
\text { Ischemia } \\
\text { Ischemia }\end{array}$ & $\begin{array}{r}\overline{10} \\
10 \\
30 \\
5\end{array}$ \\
\hline 9 & 66 & $\mathrm{~F}$ & $\begin{array}{l}\text { 1) Hypertensive } \\
\text { vascular } \\
\text { disease } \\
\text { (unknown } \\
\text { duration) } \\
\text { 2) Old cerebro- } \\
\text { vascular } \\
\text { accident }\end{array}$ & $\begin{array}{l}-\overline{.04} \\
.06 \\
.09 \\
.11\end{array}$ & $\begin{array}{l}212 / 106 \\
220 / 108 \\
232 / 100 \\
244 / 104 \\
258 / 110\end{array}$ & $\begin{array}{l}72 \\
72 \\
72 \\
72 \\
72\end{array}$ & $\begin{array}{c}2 \times \\
3 \times \\
\text { Initial ischemia } \\
\text { Ischemia } \\
\text { Ischemia }\end{array}$ & $\begin{array}{r}- \\
5 \\
5 \\
10 \\
55\end{array}$ \\
\hline
\end{tabular}

* Average number of constrictions of the arterial segments of the capillary loops per minute. $0=0-2, \times=3-8$, $1 \times=9-14,2 \times=15-25,3 \times=25-30$. 
TABLE II-Continued

\begin{tabular}{|c|c|c|c|c|c|c|c|c|}
\hline $\begin{array}{l}\text { Patient } \\
\text { no. }\end{array}$ & Age & Sex & Diagnosis & $\begin{array}{c}\text { Rate of } \\
\text { norepinephrine } \\
\text { infusion } \\
(\text { gamma/Kg./minute })\end{array}$ & $\begin{array}{l}\text { Arterial } \\
\text { blood } \\
\text { pressure } \\
(\boldsymbol{m m} . \mathrm{Hg})\end{array}$ & $\begin{array}{l}\text { Pulse } \\
\text { rate } \\
\text { (per } \\
\text { minute) }\end{array}$ & $\begin{array}{c}\text { Intensity of } \\
\text { vasomotion of } \\
\text { the capillary bed } \\
\text { of nailfold* }\end{array}$ & $\begin{array}{c}\text { Duration } \\
\text { of } \\
\text { infusion } \\
\text { (minutes) }\end{array}$ \\
\hline 10 a) & 52 & M & $\begin{array}{l}\text { 1) Hypertensive } \\
\text { vascular } \\
\text { disease } \\
\text { (known 6years) } \\
\text { 2) Old cerebral } \\
\text { thrombosis }\end{array}$ & $\begin{array}{l}-.06 \\
.08 \\
.12 \\
.19 \\
.24\end{array}$ & $\begin{array}{l}210 / 110 \\
210 / 110 \\
210 / 116 \\
220 / 116 \\
220 / 118 \\
234 / 120\end{array}$ & $\begin{array}{l}88 \\
88 \\
88 \\
80 \\
80 \\
80\end{array}$ & $\begin{array}{c}\text { Xx } \\
\text { Initial ischemia } \\
\text { Ischemia } \\
\text { Ischemia } \\
\text { Ischemia }\end{array}$ & $\begin{array}{l}-10 \\
10 \\
10 \\
10 \\
10\end{array}$ \\
\hline b) & & & $\begin{array}{l}\text { Same patient } \\
10 \text { days later }\end{array}$ & $\begin{array}{l}-.09 \\
.15 \\
.25\end{array}$ & $\begin{array}{l}190 / 102 \\
200 / 100 \\
200 / 110 \\
246 / 130\end{array}$ & $\begin{array}{l}88 \\
88 \\
84 \\
76\end{array}$ & $\begin{array}{c}2 \times \\
\text { Initial ischemia } \\
\text { Ischemia } \\
\text { Ischemia }\end{array}$ & $\begin{array}{l}-10 \\
10 \\
10\end{array}$ \\
\hline 11 & 60 & $\mathrm{~F}$ & $\begin{array}{l}\text { 1) Hypertensive } \\
\text { vascular } \\
\text { disease } \\
\text { (known } 5 \text { years) } \\
\text { 2) Acute gastritis } \\
\text { (subsided) }\end{array}$ & $\begin{array}{l}- \\
.03 \\
.05 \\
.09 \\
.13 \\
.17 \\
.28\end{array}$ & $\begin{array}{l}210 / 110 \\
210 / 110 \\
226 / 110 \\
220 / 110 \\
220 / 110 \\
220 / 116 \\
236 / 116\end{array}$ & $\begin{array}{r}100 \\
88 \\
88 \\
88 \\
88 \\
88 \\
88\end{array}$ & $\begin{array}{c}3 \times \\
\text { Initial ischemia } \\
\text { Ischemia } \\
\text { Ischemia } \\
\text { Ischemia } \\
\text { Ischemia } \\
\text { Ischemia }\end{array}$ & $\begin{array}{l}- \\
15 \\
15 \\
10 \\
10 \\
10 \\
10\end{array}$ \\
\hline 12 & 52 & F & $\begin{array}{l}\text { Hypertensive } \\
\text { vascular } \\
\text { disease. } \\
\text { (unknown } \\
\text { duration) }\end{array}$ & $\begin{array}{l}-03 \\
.03 \\
.07 \\
.09 \\
.11 \\
.15\end{array}$ & $\begin{array}{l}210 / 120 \\
226 / 120 \\
240 / 118 \\
250 / 130 \\
250 / 134 \\
260 / 130\end{array}$ & $\begin{array}{l}80 \\
72 \\
72 \\
72 \\
72 \\
72\end{array}$ & $\begin{array}{c}2 \times \\
3 \times \\
3 \times \\
\text { Initial ischemia } \\
\text { Ischemia } \\
\text { Ischemia }\end{array}$ & $\begin{array}{l}- \\
10 \\
10 \\
10 \\
10 \\
15\end{array}$ \\
\hline 13 & 66 & $\mathrm{~F}$ & $\begin{array}{l}\text { 1) Hypertensive } \\
\text { vascular } \\
\text { disease } \\
\text { (known } 3 \text { years) } \\
\text { 2) Osteoarthritis }\end{array}$ & $\begin{array}{l}\overline{.05} \\
.10\end{array}$ & $\begin{array}{l}240 / 130 \\
260 / 134 \\
270 / 136\end{array}$ & $\begin{array}{l}84 \\
84 \\
84\end{array}$ & $\begin{array}{c}3 \times \\
\text { Initial ischemia } \\
\text { Ischemia }\end{array}$ & $\begin{array}{l}- \\
10 \\
15\end{array}$ \\
\hline 14 & 57 & $F$ & $\begin{array}{l}\text { 1) Hypertensive } \\
\text { vascular } \\
\text { disease } \\
\text { (known } 15 \text { years) } \\
\text { 2) Cerebrovascular } \\
\text { accident }\end{array}$ & $\begin{array}{l}-\overline{.08} \\
.14 \\
.23\end{array}$ & $\begin{array}{l}220 / 132 \\
236 / 138 \\
238 / 140 \\
240 / 140\end{array}$ & $\begin{array}{l}100 \\
100 \\
100 \\
100\end{array}$ & $\begin{array}{c}3 \times \\
\text { Initial ischemia } \\
\text { Ischemia } \\
\text { Ischemia }\end{array}$ & $\begin{array}{l}\overline{10} \\
15 \\
15\end{array}$ \\
\hline 15 & 38 & $\mathrm{~F}$ & $\begin{array}{l}\text { Hypertensive } \\
\text { vascular } \\
\text { disease } \\
\text { (known } 5 \text { years) }\end{array}$ & $\begin{array}{l}-\overline{.08} \\
.16 \\
.32\end{array}$ & $\begin{array}{l}240 / 140 \\
240 / 140 \\
250 / 140 \\
250 / 144\end{array}$ & $\begin{array}{l}84 \\
84 \\
84 \\
80\end{array}$ & $\begin{array}{l}3 \times \\
\text { Initial ischemia } \\
\text { Ischemia } \\
\text { Ischemia }\end{array}$ & $\begin{array}{l}\overline{15} \\
15 \\
20\end{array}$ \\
\hline
\end{tabular}

laries. Epinephrine was also observed to increase the frequency of interruptions of blood flow through the terminal capillary loops of the nailfold. For these reasons the interruptions of blood flow through the capillary loops of the nailfold were interpreted as the result of irregular, periodic vasoconstriction of the minute vascular bed. The relative contribution of the arterioles, metarterioles, and arterial segments of the capillary loops in the production of these gaps in blood flow could not be determined. (The proximal portions of the arterial segments of the capillary loops probably were capable of active vasoconstriction since the interruptions in blood flow occurred independently in adjacent capillary loops.) The process of in- termittent vasoconstriction of the minute vascular bed has been termed "vasomotion" (3-5).

In patients with normal blood pressure, there were usually 3 to 14 interruptions of blood flow through the arterial segment of the capillary loops per minute (denoted in Table I under Intensity of Vasomotion by $X$ and $1 \times$ ). Each cessation of blood flow rarely exceeded two seconds in duration. In patients with essential hypertension there was usually a definite increase in the frequency of interruptions of blood flow through the capillary loops, often averaging 15 to 30 cessations of flow per minute (denoted in Table II under Intensity of Vasomotion by $2 \times$ and $3 \times$ ). The duration of each interruption in flow was as transient as that in persons with normal blood pressure. 


\section{L-nor-epinephrine infusion, normotensive subjects (Table I)}

When 1-nor-epinephrine was infused intravenously in patients with normal blood pressure, both the systolic and diastolic pressures increased as the rate of infusion increased. Concomitantly, vasomotion increased progressively as indicated by the increasing frequency of cessations of capillary blood flow. The duration of each interruption of blood flow increased but slightly, so that the resulting ischemia of the arterial segment was not maintained for intervals longer than two seconds. A stage was finally attained during which the capillary bed appeared similar to that observed in patients with essential hypertension. This stage was reached when the diastolic pressure had been elevated an average of $11 \mathrm{~mm}$. $\mathrm{Hg}$ (range from 4 to $26 \mathrm{~mm} . \mathrm{Hg}$ ) to levels of 70 to $90 \mathrm{~mm}$. $\mathrm{Hg}$ (Table I).

When the rate of 1-nor-epinephrine infusion was increased further, not only did the frequency of interruptions of blood flow through the arterial segments of the capillary loops increase, but the duration of the cessations of flow became progressively longer until an "ischemic state" developed which no longer resembled the capillary blood flow seen in patients with essential hypertension. The entire capillary loop (not the arterial segment alone) now disappeared from view for periods of two seconds to several hours. At times complete cessation of blood flow through the capillary loop occurred so that stagnated clumps of red blood

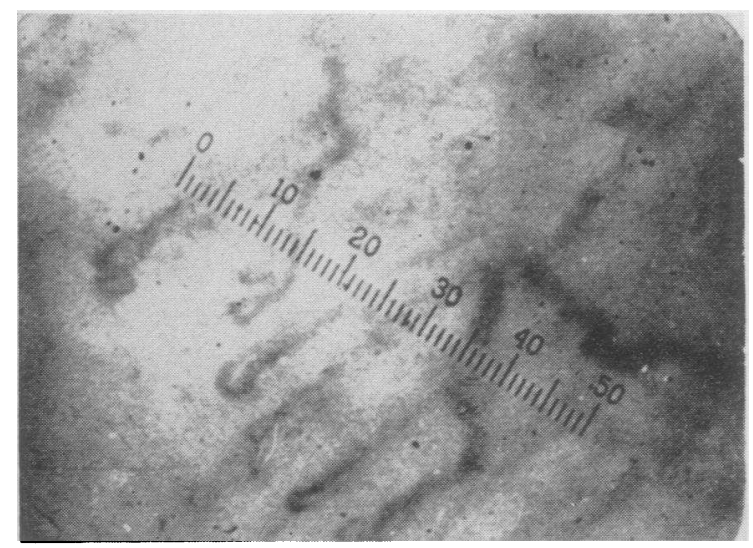

Fig. 1. Capillary Bed of the Nailfold of a Patient with Normal Blood Pressure $(68 \times)$ B.P. $=$ 126/60 P.R. $=76$

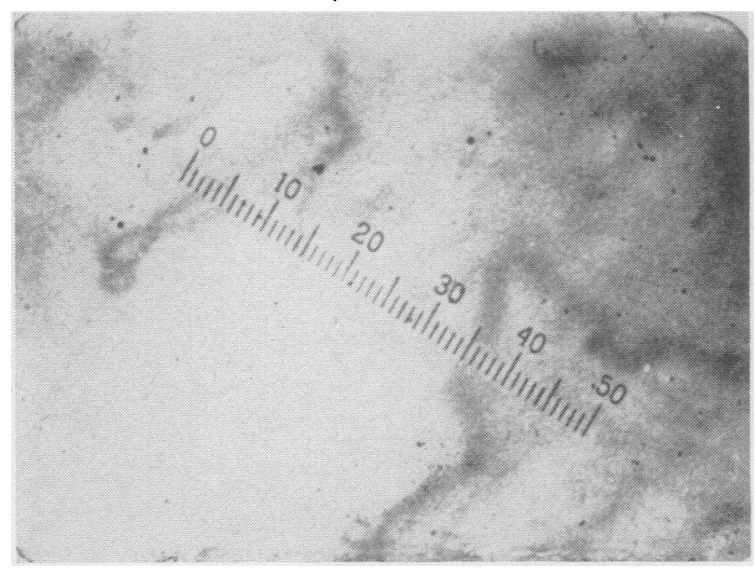

Fig. 2. Initial Ischemic Reaction of the CapilLARY Bed dURING L-Nor-epinephrine Infusion $(68 \times)$ 0.20 Gamma/KG./Minute B.P. $=130 / 70$ P.R. $=74(40$ Minutes after Start of Infusion)

cells marked the outline of the original blood vessel. The ischemic state persisted virtually unchanged as long as the infusion continued.

The ischemic reaction did not occur simultaneously in all the capillary loops. Of an average of ten loops usually visualized in any one microscopic field, ischemia occurred initially and persisted in two or three capillary loops, generally those adjacent to each other and of the narrowest caliber. As the rate of infusion was increased, increasing numbers of capillary vessels reacted similarly. The point at which ischemia occurred in two or three of the capillary loops in one microscopic field was selected as the point of "initial ischemic reaction." In persons with normal cardiovascular systems, initial ischemia of the capillary bed of the nailfold developed when the rate of 1-nor-epinephrine infusion raised the diastolic pressure an average of 17 $\mathrm{mm} . \mathrm{Hg}$ (range from 10 to $30 \mathrm{~mm} . \mathrm{Hg}$ ) to levels of 80 to $100 \mathrm{~mm}$. Hg. The ischemic reaction persisted virtually unchanged for the duration of the infusion, twenty minutes to three hours. Capillary ischemia was not usually seen in patients with essential hypertension, even at diastolic pressure levels of $140 \mathrm{~mm}$. $\mathrm{Hg}$.

In fifteen patients with normal blood pressures the mean rate of infusion of 1 -nor-epinephrine required to produce an initial ischemic reaction was 0.23 gamma per $\mathrm{Kg}$. per minute (standard deviation of 0.07$)$. Figure 1 is a photomicrograph $(68 \times)$ of the capillary bed of the nailfold of a patient with normal blood pressure. Figure 2 
indicates the initial ischemic reaction of the capillary bed during the infusion of 1-nor-epinephrine.

\section{L-nor-epinephrine infusion, hypertensive patients (Table II)}

When l-nor-epinephrine was infused in patients with essential hypertension, both the systolic and diastolic pressures increased as the rate of infusion was increased. The rise in systolic pressure with low rates of infusion was significantly greater than the rise which occurred in patients with normal blood pressures (Table III). As the rate of infusion was increased, however, the rise of systolic pressure became progressively less marked, as compared to the continuing rise of systolic pressure in patients with normal blood pressures. There was no significant difference in the rate of elevation of the diastolic or mean arterial pressures in patients with essential hypertension and with normal blood pressures (Table III).
As 1-nor-epinephrine was infused in patients with hypertensive vascular disease, vasomotion of the minute vessel bed, initially greater than normal, increased so rapidly that an ischemic reaction occurred with low rates of infusion (Table II). Again the "disappearance" of capillary loops and the stagnation of clumps of red blood cells in adjacent vessels did not occur simultaneously. Usually two or three loops in any one microscopic field were involved initially. As the rate of infusion was increased, progressively more capillary loops became ischemic. In patients with essential hypertension, the initial ischemic reaction of the capillary bed occurred when the rate of the 1-norepinephrine infusion had raised the diastolic pressure an average of $4 \mathrm{~mm}$. $\mathrm{Hg}$ (range from 0 to 18 $\mathrm{mm} . \mathrm{Hg}$ ) from the initial level. In fifteen patients with essential hypertension, the mean rate of infusion of 1-nor-epinephrine required to produce an initial ischemic reaction of the capillary bed

TABLE III

Resting levels and increases of systemic arterial pressures during l-nor-epinephrine infusion in patients with normal blood pressure and with essential hypertension

\begin{tabular}{|c|c|c|c|c|c|c|c|c|}
\hline & \multicolumn{4}{|c|}{ Normal blood pressure } & \multicolumn{4}{|c|}{ Essential hypertension } \\
\hline & $\begin{array}{l}\text { No. } \\
\text { cases }\end{array}$ & Mean & $\begin{array}{l}\text { Standard } \\
\text { deviation }\end{array}$ & Range & $\begin{array}{l}\text { No. } \\
\text { cases }\end{array}$ & Mean & $\begin{array}{l}\text { Standard } \\
\text { deviation }\end{array}$ & Range \\
\hline \multicolumn{9}{|c|}{ I. Resting levels } \\
\hline 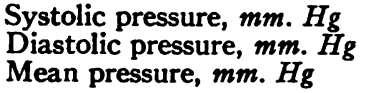 & $\begin{array}{l}15 \\
15 \\
15\end{array}$ & $\begin{array}{r}122 \\
70 \\
90\end{array}$ & $\begin{array}{l}13 \\
10 \\
10\end{array}$ & $\begin{array}{l}100-142 \\
54-90 \\
76-106\end{array}$ & $\begin{array}{l}15 \\
15 \\
15\end{array}$ & $\begin{array}{l}200 \\
105 \\
141\end{array}$ & $\begin{array}{l}31 \\
16 \\
22\end{array}$ & $\begin{array}{r}146-240 \\
86-140 \\
110-180\end{array}$ \\
\hline
\end{tabular}

II. Increase of pressures with increasing doses of l-nor-epinephrine

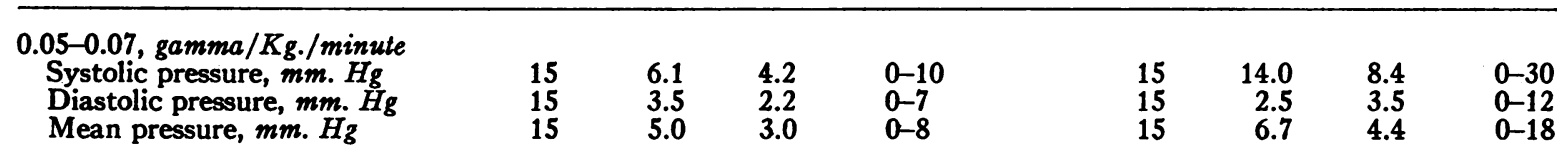

( $t$ for systolic blood pressure changes in patients with normal blood pressures as compared with those of essential hypertension $=3.2$ )

$0.10-0.13$, gamma $/ \mathrm{Kg}$. minute Systolic pressure, $m m$. $H g$

Diastolic pressure, $\mathrm{mm}$. $\mathrm{Hg}$

Mean pressure, $\mathrm{mm}$. $\mathrm{Hg}$

$\begin{array}{rrrrrrrr}15 & 16.0 & 12.0 & 0-40 & 15 & 24.0 & 15.4 & 5-60 \\ 15 & 8.8 & 5.0 & 4-20 & 15 & 5.5 & 4.5 & 0-18 \\ 15 & 12.0 & 5.8 & 4-22 & 15 & 13.8 & 8.7 & 2-30\end{array}$

( $t$ for systolic blood pressure changes in patients with normal blood pressures as compared with those of essential hypertension $=1.5$ )

\subsection{6-0.20, gamma/Kg./minute} Systolic pressure, $\mathrm{mm}$. $\mathrm{Hg}$ Diastolic pressure, $m m$. $H_{g}$ Mean pressure, $m m$. $H g$

$\begin{array}{rrrrrrrr}15 & 30.0 & 15.4 & 4-56 & 13 & 35.0 & 20.6 & 10-70 \\ 15 & 14.7 & 5.5 & 6-25 & 13 & 11.4 & 8.0 & 0-26 \\ 15 & 21.5 & 7.6 & 8-36 & 13 & 20.6 & 11.0 & 2-34\end{array}$


was 0.07 gamma per $\mathrm{Kg}$. per minute (standard deviation of 0.025 ).

\section{DISCUSSION}

The total systemic peripheral blood flow in patients with essential hypertension is not significantly different from the blood flow in persons with normal blood pressure $(6,7)$. In one aspect of the peripheral blood flow, the capillary circulation of the nailfold of the finger, a specific difference in the hypertensive patient is noted. Vasomotion, the irregular momentary cessations of capillary blood flow which probably reflects periodic, irregular vasoconstriction of the contractile elements of the minute vascular bed, is usually accelerated in patients with essential hypertension. The infusion of 1-nor-epinephrine appeared to intensify vasomotion. When 1-nor-epinephrine was infused intravenously in persons with normal blood pressures, the capillary circulation of the nailfold began to resemble that observed in patients with essential hypertension. There was, however, a significant difference. Vasomotion of the capillary bed to a degree resembling that seen in patients with essential hypertension was produced by l-norepinephrine when the diastolic blood pressure levels were still within the normal range. Attempts to increase the diastolic blood pressure to levels found in patients with essential hypertension produced a progressive and persistent ischemia of the capillary bed, an appearance not usually seen in patients with essential hypertension. Apparently the diastolic hypertensive state associated with the infusion of 1-nor-epinephrine may have central systemic hemodynamics similar to those observed in the diastolic hypertensive state associated with essential hypertension but not a similar nailfold capillary circulation.

The capillary bed of the nailfold of patients with essential hypertension appeared significantly more reactive to 1 -nor-epinephrine than the same capillary bed in patients with normal blood pressure, as judged by the rates of infusion required to produce initial ischemia of the capillaries $(t=$ 8.4). This could not be attributed solely to the increased intensity of vasomotion initially present in persons with essential hypertension whereby only a slight additional vasoconstriction induces an ischemic reaction. As indicated in Table II, vasomotion in patients number 3 and $10 \mathrm{a}$ was not more intense initially than in the average patient with a normal blood pressure. The quantity of 1-nor-epinephrine required to induce ischemia, however, fell in the same range as that required by hypertensive patients with initially increased vasomotion. This hyper-reactivity of the capillary bed of the nailfold of patients with essential hypertension to sustained intravenous infusions of 1-norepinephrine conforms with the previously reported hyper-reactivity to single injections (2).

The reaction of the circulation in the capillary bed of the nailfold proved to be a more sensitive index for detecting small quantities of intravenously injected l-nor-epinephrine than was the reaction of the arterial blood pressure, as determined by the auscultatory method. Striking alterations in the capillary blood flow during the lower rates of 1-nor-epinephrine infusion were often accompanied by absent or minor alterations in the blood pressure (Table I and II). This was especially noted in patients with essential hypertension.

The elevations in blood pressure and the effects induced in the capillary bed of the nailfold by the infusion of 1-nor-epinephrine represented acute changes, for periods not exceeding three hours. During this period, the capillary bed exhibited few signs of adaptation. Even during marked ischemia there was no tendency to return toward the normal state as long as the infusion of 1-nor-epinephrine continued at a steady rate. It is possible, however, that 1-nor-epinephrine, acting over an extended period, could produce a chronic hypertensive state without inducing a chronic ischemic reaction in the capillary bed of the nailfold. A study of the capillary bed in patients with persistent hypertension due to pheochromocytoma would, therefore, be of interest.

It would be tempting to conclude that the reactions of the capillary circulation of the nailfold here reported indicate the reactivity of the capillary circulation as a whole. In support of such a conclusion one might cite observations which indicate that the reactions of the capillaries of two other areas, the mesoappendix of the rat (8) and the bulbar conjunctivae of man (9), are similar to those here noted for the nailfold. However, a conclusion that the systemic capillary circulation as a whole is more reactive in 1-nor-epinephrine induced hypertension than in essential hypertension 
is specifically avoided. The variations in capillary reactivity in different vascular areas are too great to permit generalizations from observations on restricted capillary fields. Accordingly, the conclusion of this study remains that for comparable levels of diastolic blood pressure the capillary circulation of the nailfold of the finger is more reactive during 1-nor-epinephrine induced hypertension than in essential hypertension. Furthermore the present observations were made in patients of the middle and older age groups with associated disease states. The results, therefore, are not necessarily applicable to young healthy adults or to young adults with uncomplicated essential hypertension.

\section{CONCLUSIONS}

1. The circulation through the terminal capillary loops of the nailfold is interrupted by irregular, momentary cessations of blood flow which probably reflect periodic, irregular vasoconstriction of the contractile portions of the minute vascular bed. This process of vasomotion is usually intensified in patients with essential hypertension.

2. L-nor-epinephrine infused intravenously at increasing rates in persons with normal blood pressures induced progressively increasing vasomotion until the capillary bed resembled that seen in patients with essential hypertension. At this stage, the diastolic blood pressure increased an average of $11 \mathrm{~mm}$. $\mathrm{Hg}$ but usually remained within normal limits.

3. Further increase in the rate of infusion of 1-nor-epinephrine induced such marked vasomotion that the capillary loops became ischemic. Initial ischemia appeared and persisted when the diastolic blood pressure was elevated an average of $17 \mathrm{~mm}$. $\mathrm{Hg}$, although the diastolic blood pressure at this stage usually did not exceed $100 \mathrm{~mm}$. $\mathrm{Hg}$.

4. The capillary bed of patients with essential hypertension was hyper-reactive to circulating 1-nor-epinephrine, as judged by the rates of infusion required to induce initial ischemia. Capillary ischemia appeared when the diastolic blood pressure was elevated by an average of only $4 \mathrm{~mm}$. $\mathrm{Hg}$.
5. The diastolic hypertensive states associated with essential hypertension and with the infusion of 1-nor-epinephrine may have similar central systemic hemodynamics but do not have similar nailfold capillary circulations.

6. The alteration of the circulation in the capillary bed of the nailfold was a more sensitive index for detecting small quantities of intravenously injected 1-nor-epinephrine than was the alteration of the arterial blood pressure, as determined by the auscultatory method.

7. The results and conclusions apply only to acute changes produced in the circulation through the capillary bed of the nailfold and in the systemic blood pressure by the intravenous infusion of 1-nor-epinephrine.

\section{REFERENCES}

1. Goldenberg, M., Pines, K. L., Baldwin, E. de F., Greene, D. G., and Roh, C. E., The hemodynamic response of man to nor-epinephrine and epinephrine and its relation to the problem of hypertension. Am. J. Med., 1948, 5, 792.

2. Greisman, S. E., The reactivity of the capillary bed of the nailfold to circulating epinephrine and norepinephrine in patients with normal blood pressure and with essential hypertension. J. Clin. Invest., 1952, 31, 782.

3. Zweifach, B. W., The character and distribution of the blood capillaries. Anat. Rec., 1939, 73, 475.

4. Chambers, R., and Zweifach, B. W., Topography and function of the mesenteric capillary circulation. Am. J. Anat., 1944, 75, 173.

5. Chambers, R., and Zweifach, B. W., Functional activity of the blood capillary bed, with special reference to visceral tissue. Ann. New York Acad. Sc., 1946, 46, 683.

6. Prinzmetal, M., and Wilson, C., The nature of the peripheral resistance in arterial hypertension with special reference to the vasomotor system. J. Clin. Invest., 1936, 15, 63.

7. Pickering, G. W., The peripheral resistance in persistent arterial hypertension. Clin. Sc., 1936, 2, 209.

8. Zweifach, B. W., Rosenfeld, S., and Shorr, E., Hepatorenal factors in circulatory homeostasis. XVI : Vascular changes in mesentery in renal hypertension in rats. Federation Proc., 1948, 7, 139.

9. Lee, R. E., and Holze, E. A., Peripheral vascular hemodynamics in the bulbar conjunctiva of subjects with hypertensive vascular disease. J. Clin. Invest., 1951, 30, 539. 